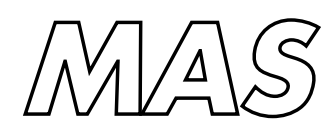

Modelling, Analysis and Simulation

\footnotetext{
Modelling, Analysis and Simulation

MAS Fix for Solution Errors near Interfaces in Two-Fluid Flow Computations

B. Koren, E.H. van Brummelen, P.W. Hemker,

B. van Leer, M.R. Lewis
} 
CWI is the National Research Institute for Mathematics and Computer Science. It is sponsored by the Netherlands Organization for Scientific Research (NWO).

CWI is a founding member of ERCIM, the European Research Consortium for Informatics and Mathematics.

CWI's research has a theme-oriented structure and is grouped into four clusters. Listed below are the names of the clusters and in parentheses their acronyms.

Probability, Networks and Algorithms (PNA)

Software Engineering (SEN)

Modelling, Analysis and Simulation (MAS)

Information Systems (INS)

Copyright (C) 2003, Stichting Centrum voor Wiskunde en Informatica

P.O. Box 94079, 1090 GB Amsterdam (NL)

Kruislaan 413, 1098 SJ Amsterdam (NL)

Telephone +31205929333

Telefax +31205924199

ISSN 1386-3703 


\title{
Fix for Solution Errors near Interfaces in Two-Fluid Flow Computations
}

\begin{abstract}
A finite-volume method is considered for the computation of flows of two compressible, immiscible fluids at very different densities. A level-set technique is employed to distinguish between the two fluids. A simple ghost-fluid method is presented as a fix for the solution errors ('pressure oscillations') that may occur near two-fluid interfaces when applying a capturing method. Computations with it for compressible two-fluid flows with arbitrarily large density ratios yield perfectly sharp, pressure-oscillation-free interfaces. The masses of the separate fluids appear to be conserved up to first-order accuracy.
\end{abstract}

2000 Mathematics Subject Classification: 76-xx

Keywords and Phrases: two-fluid flow; ghost-fluid method 


\title{
Fix for Solution Errors near Interfaces in Two-Fluid Flow Computations
}

\author{
B. Koren ${ }^{1}$, E.H. van Brummelen ${ }^{1}$, P.W. Hemker ${ }^{1}$, B. van Leer $^{2}$, and \\ M.R. Lewis ${ }^{1}$ \\ 1 CWI, P.O. Box 94079, 1090 GB Amsterdam, The Netherlands \\ 2 The University of Michigan, Ann Arbor, MI 48109-2140, USA
}

\begin{abstract}
A finite-volume method is considered for the computation of flows of two compressible, immiscible fluids at very different densities. A level-set technique is employed to distinguish between the two fluids. A simple ghost-fluid method is presented as a fix for the solution errors ('pressure oscillations') that may occur near two-fluid interfaces when applying a capturing method. Computations with it for compressible two-fluid flows with arbitrarily large density ratios yield perfectly sharp, pressureoscillation-free interfaces. The masses of the separate fluids appear to be conserved up to first-order accuracy.
\end{abstract}

\section{Introduction}

A known difficulty of capturing two-fluid interfaces in a conservative formulation of the compressible Euler equations is that $\mathcal{O}\left(h^{0}\right)=\mathcal{O}(1)$ solution errors (in literature often called 'pressure oscillations') may arise near the two-fluid interface. Without remedial intervening, the conservative formulation considered in the present paper also suffers from the pressure-oscillation problem. Fixes for the problem can be found in the literature. We refer to the works of Karni [6,7] and Abgrall [1], their common paper [2], and also to [3,5,9]. In most of the available literature though, the ratio of the two densities at the interface is $\mathcal{O}(1)-\mathcal{O}\left(10^{2}\right)$. To our knowledge, only in $[3,9]$ ratios of $\mathcal{O}\left(10^{3}\right)$, typical water-air ratios, are considered. In this paper a fix is proposed, which allows arbitrarily large density ratios. The fix is a simple variant of the ghost-fluid method [3].

\section{Flow Model}

\subsection{Conservation Equations}

In $1 \mathrm{D}$, for a sufficiently small control volume $\Omega$, conservation of mass and momentum reads:

$\int_{\Omega} \frac{\partial \boldsymbol{q}}{\partial t} \mathrm{~d} x+\boldsymbol{f}(\boldsymbol{q})_{\partial \Omega_{\mathrm{right}}}-\boldsymbol{f}(\boldsymbol{q})_{\partial \Omega_{\mathrm{left}}}=0, \quad \boldsymbol{q}=\left(\begin{array}{c}\varrho \\ \varrho u\end{array}\right), \quad \boldsymbol{f}(\boldsymbol{q})=\left(\begin{array}{c}\varrho u \\ \varrho u^{2}+p\end{array}\right)$,

with $\varrho$ the bulk density: $\varrho=\alpha(x, t) \varrho_{w}(p)+(1-\alpha(x, t)) \varrho_{a}(p)$, where $\alpha$ is, e.g., the volume-of-water fraction, and where $\varrho_{w}(p)$ and $\varrho_{a}(p)$ are the equations of 
state for water and air, respectively. To balance this system, the equations of state and an equation determining the location of the interface (and hence $\alpha$ ) still have to be chosen. An accurate resolution of the interface location(s) is of paramount importance. For this purpose, we follow a level-set approach, to be outlined in the next section.

\subsection{Level-Set Equation}

To accurately resolve the interface location(s), a level-set approach [10] is more appropriate than the classical volume-of-fluid (VOF) approach [4], because of its better smoothness (and thus accuracy) properties at precisely the point of interest: the interface. Good smoothness of the level-set function is first taken care of in the level-set function's initialization. A common approach is to initialize the level-set function as the signed distance to the initial interface, with the distance positive in, e.g., water and negative in air. During the computation, the level-set function may need to be reinitialized.

Denoting the level-set function by $\phi$, in $1 \mathrm{D}$, it is advected: $\frac{\partial \phi}{\partial t}+u \frac{\partial \phi}{\partial x}=0$. Combined with the bulk-mass conservation equation from (1), this advection equation may be written in the conservative control-volume form

$$
\int_{\Omega} \frac{\partial(\varrho \phi)}{\partial t} \mathrm{~d} x+(\varrho u \phi)_{\partial \Omega_{\mathrm{right}}}-(\varrho u \phi)_{\partial \Omega_{\mathrm{left}}}=0 .
$$

Conservation of $\varrho \phi$ is not physical, there is no conservation law for it. The form (2) is simply practical because it is consistent with system (1).

\subsection{Equation of State}

In homentropic water-air computations, for both fluids, elegant use can be made of a single equation of state, viz. Tait's: $\frac{p+B p_{\text {ref }}}{(1+B) p_{\text {ref }}}=\left(\frac{\varrho}{\varrho_{\text {ref }}}\right)^{\gamma}$, where the subscript 'ref' indicates some reference state. The reference pressure $p_{\text {ref }}$ is chosen freely but equally for the two fluids. The value of $\varrho_{\text {ref }}$ for each of the two fluids corresponds with $p_{\text {ref }}$ and is read from standard data bases for fluid properties. Concerning the material constants $\gamma$ and $B$, for water it holds: $\gamma=7, B=3000$ and for air: $\gamma=\frac{7}{5}, B=0$.

\section{Ghost-Fluid Method}

In [8], it is shown that - unfortunately - the present conservative formulation (1)-(2) also leads to $\mathcal{O}(1)$ pressure errors near two-fluid interfaces. As a remedy against this, we outline a simple variant of the ghost-fluid method.

In updating the finite-volume solutions with a single explicit time step the following is done. Suppose we have an equidistant, cell-centered finite-volume grid $\Omega_{i}, i=1,2, \ldots, N$, with cell faces $\partial \Omega_{i+\frac{1}{2}}, i=0,1, \ldots, N$, where $\partial \Omega_{\frac{1}{2}}$ and $\partial \Omega_{N+\frac{1}{2}}$ are at the domain boundaries. Also suppose that at time level $n$ we 
have a known, unique solution $\left(u_{i}^{n}, p_{i}^{n}, \phi_{i}^{n}\right), i=1,2, \ldots, N$. Then, at first, at the actual time level $n$ the cells and cell faces are classified into different types. For cells, the following three types are distinguished: (i) pure-water cells, (ii) pureair cells and (iii) cells with one (or two) interface(s). To make this classification, we determine $\phi_{i+\frac{1}{2}}^{n}=\frac{1}{2}\left(\phi_{i}^{n}+\phi_{i+1}^{n}\right), i=1,2, \ldots, N-1$. At the inflow-boundary face, say $\partial \Omega_{\frac{1}{2}}$, we take $\phi_{\frac{1}{2}}^{n}=\phi_{b}^{n}$, with $\phi_{b}^{n}$ the boundary condition, and at the outflow boundary, say $\partial \Omega_{N+\frac{1}{2}}: \phi_{N+\frac{1}{2}}^{n}=\phi_{N}$. Then, cell $\Omega_{i}$ is: (i) a pure-water cell if $\phi_{i}^{n}>0, \phi_{i-\frac{1}{2}}^{n}>0$ and $\phi_{i+\frac{1}{2}}^{n}>0$, (ii) a pure-air cell if $\phi_{i}^{n}<0, \phi_{i-\frac{1}{2}}^{n}<0$ and $\phi_{i+\frac{1}{2}}^{n}<0$, and - else - (iii) a cell with one or two interfaces. The third type of cells are named ghost cells. This classification is also applied to the cell faces; (i) pure-water, (ii) pure-air and (iii) ghost faces are distinguished. The two faces of a ghost cell are both identified as ghost faces. I.e., if $\Omega_{i}$ is found to be a ghost cell, then both $\partial \Omega_{i-\frac{1}{2}}$ and $\partial \Omega_{i+\frac{1}{2}}$ are ghost faces. A cell face not belonging to a ghost cell is - depending on the sign of $\phi$ at that face - either a pure-water or a pure-air face. Across the latter two types of faces, the flux is simply computed with the single-fluid linearized Godunov scheme $\boldsymbol{F}\left(\tilde{\boldsymbol{q}}_{0}, \tilde{\boldsymbol{q}}_{1}\right), \tilde{\boldsymbol{q}} \equiv(u, p)$. So, across pure-water faces we get $\boldsymbol{F}=\boldsymbol{F}_{w}$ and across pure-air faces $\boldsymbol{F}=\boldsymbol{F}_{a}$. Across the ghost faces two fluxes are computed: a water and an air flux, so both $\boldsymbol{F}_{w}\left(\tilde{\boldsymbol{q}}_{0}, \tilde{\boldsymbol{q}}_{1}\right)$ and $\boldsymbol{F}_{a}\left(\tilde{\boldsymbol{q}}_{0}, \tilde{\boldsymbol{q}}_{1}\right)$. Applying the forward Euler scheme, the subsequent update of finite-volume solutions reads then:

(i) in pure-water cells: $\boldsymbol{q}_{i}^{n+1}=\boldsymbol{q}_{i}^{n}-\frac{\Delta t}{h}\left(\left(\boldsymbol{F}_{w}\right)_{i+\frac{1}{2}}^{n}-\left(\boldsymbol{F}_{w}\right)_{i-\frac{1}{2}}^{n}\right)$,

(ii) in pure-air cells: $\boldsymbol{q}_{i}^{n+1}=\boldsymbol{q}_{i}^{n}-\frac{\Delta t}{h}\left(\left(\boldsymbol{F}_{a}\right)_{i+\frac{1}{2}}^{n}-\left(\boldsymbol{F}_{a}\right)_{i-\frac{1}{2}}^{n}\right)$,

(iii) in ghost cells: $\left(\boldsymbol{q}_{w}\right)_{i}^{n+1}=\boldsymbol{q}_{i}^{n}-\frac{\Delta t}{h}\left(\left(\boldsymbol{F}_{w}\right)_{i+\frac{1}{2}}^{n}-\left(\boldsymbol{F}_{w}\right)_{i-\frac{1}{2}}^{n}\right)$ and

$$
\left(\boldsymbol{q}_{a}\right)_{i}^{n+1}=\boldsymbol{q}_{i}^{n}-\frac{\Delta t}{h}\left(\left(\boldsymbol{F}_{a}\right)_{i+\frac{1}{2}}^{n}-\left(\boldsymbol{F}_{a}\right)_{i-\frac{1}{2}}^{n}\right) .
$$

So, in ghost cells we are left with two, possibly different updated solutions: $\boldsymbol{q}_{w}$ and $\boldsymbol{q}_{a}$. Expressed in $\tilde{\boldsymbol{q}}=(u, p)$-variables, these two new ghost solutions will not differ very much for the flows considered here. In case a solution ambiguity does arise, we proceed as follows. From the updated level-set function, which is updated separately through an advection equation, the volume-of-fluid fraction $\alpha_{i}^{n+1}$ in the ghost cell is computed. Next, the updated solution in the ghost cell is made unique with

$$
\tilde{\boldsymbol{q}}_{i}^{n+1}=\alpha_{i}^{n+1}\left(\tilde{\boldsymbol{q}}_{w}\right)_{i}^{n+1}+\left(1-\alpha_{i}^{n+1}\right)\left(\tilde{\boldsymbol{q}}_{a}\right)_{i}^{n+1}
$$

\section{Numerical Results Ghost-Fluid Method}

\subsection{Water Front at Constant Speed and Pressure}

Consider a $1 \mathrm{D}$ tube with unit length, $x \in[0,1]$, inflow at $x=0$, outflow at $x=1$ and with as initial solution: $u(x, t=0)=1, p(x, t=0)=1,\left(x_{\mathrm{fs}}\right)_{t=0}=0, \varrho(x=$ $0, t=0)=\varrho_{w}(1)=1$, and $\varrho(x, t=0)_{x>0}=\varrho_{a}(1) \ll 1$, where $x_{\mathrm{fs}}$ is the location 
of the free surface, i.e., the water-air interface. The boundary conditions to be imposed are $u(x=0, t)=1, \phi(x=0, t)=t$ and $p(x=1, t)=1$. The exact Eulerflow solution reads $u(x, t)=1, p(x, t)=1, \varrho(x, t)_{x<t}=1$ and $\varrho(x, t)_{x>t}=\varrho_{a}(1)$. This simple model flow precisely uncovers the deficiency of capturing methods with regard to material interfaces. Various values are considered for the density ratio $\frac{\varrho_{w}}{\varrho_{a}}$. The grids to be used are equidistant. The space discretization is taken first-order accurate and time integration is done with the forward Euler scheme.

Results of several numerical approaches that do not work satisfactorily are given in [8]. The ghost-fluid method performs very well; pressure (not shown) remains constant. In Figure 1 we present the computed bulk-density profiles at $t=0.0,0.1,0.2, \ldots, 1.0$. The results are perfect; the interface is captured over a single mesh width $h$ only (thanks to the level-set approach). The method does not break down with increasing density ratio. It works for standard water-air density ratios, $\frac{\underline{\omega}_{w}}{\varrho_{a}}=\mathcal{O}\left(10^{3}\right)$, as well as for much larger ratios (Figure 2). Reinitialization of the level-set function is not necessary for the running water front.
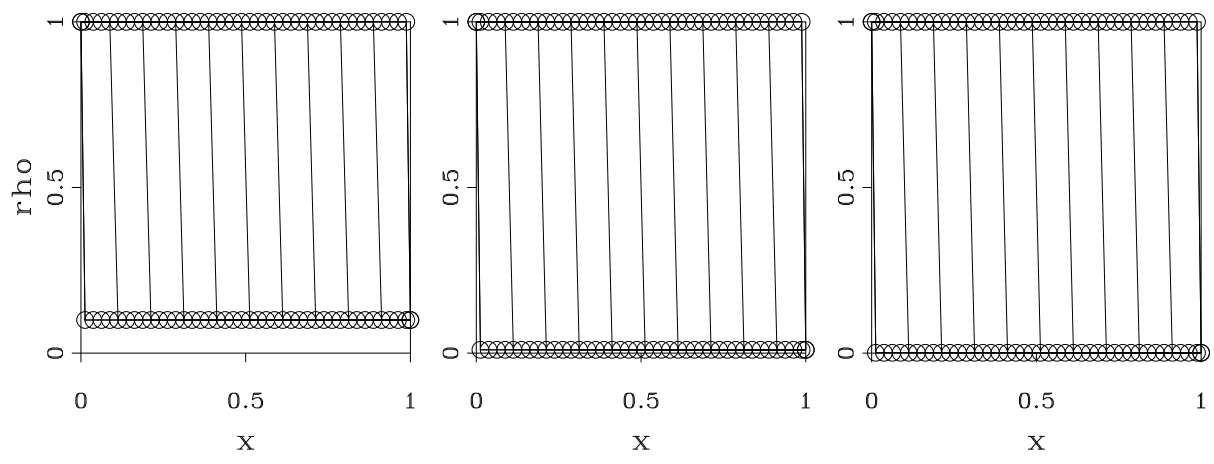

Fig. 1. Bulk-density profiles at $t=0.0,0.1,0.2, \ldots, 1.0, h=\frac{1}{40}$, density ratios from left to right: $10,100,1000$

\subsection{Oscillating Water Column}

In the ghost cells, the conservation laws are applied to virtual amounts of water and air, not to the real physical amounts. Hence, proper conservation of mass and momentum in these cells is not guaranteed. As a second test case, we consider a closed 1D tube (impermeable boundary at the left and right), with the initial condition as sketched in Figure 3. Starting from $t=0$, the air at the right is compressed by the water and the air at the left expands. The column of water is decelerated until stagnation, and next accelerated to the left. The latter leads to a reverse pressure gradient across the water column, which redirects the flow from left to right again, and so on: the water column starts to oscillate.

We present numerical results obtained through the ghost-fluid method, taking $\frac{\varrho_{w}}{\varrho_{a}}=1000$ and $\left(x_{\mathrm{fs}}\right)_{t=0}=0.1$. An equidistant grid with $h=\frac{1}{40}$ is applied. The 

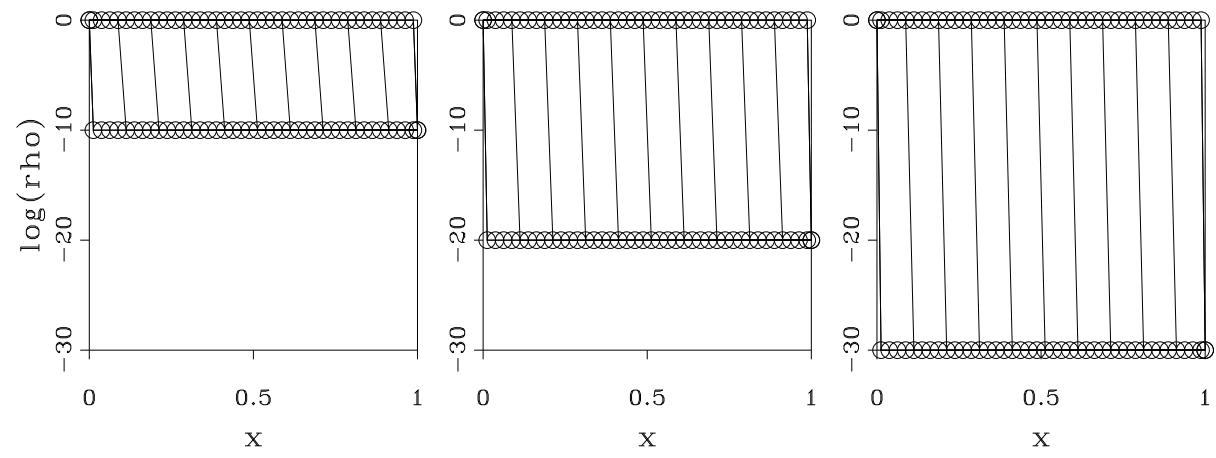

Fig. 2. Bulk-density profiles at $t=0.0,0.1,0.2, \ldots, 1.0, h=\frac{1}{40}$, density ratios from left to right: $10^{10}, 10^{20}, 10^{30}$

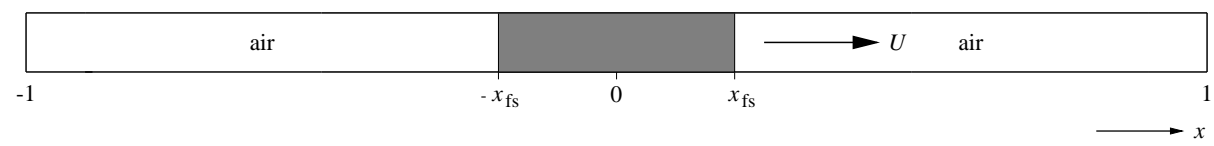

Fig. 3. Initial condition: closed tube with column of water (grey) in between two columns of air, all three columns flowing to the right at constant speed and pressure

space discretization is again first-order accurate and time integration is done again with the forward Euler scheme. The level-set function is taken as the signed-distance function. For this test case, the level-set function is reinitialized. (The reinitialization is done after each time step.)

In the left graph of Figure 4 we give the time evolution of the relative mass error $\mathcal{M}(t) \equiv \frac{m_{a}(t)-m_{a}(0)}{m_{a}(0)}$, where $m_{a}(t)$ is the total mass of air in the tube at time $t$. The error appears to be composed of two components: one oscillating and the other behaving linearly in time. The total mass of air is slowly decreasing; air is converted into water. Fortunately, the orders of both the oscillatory and the linear error component are close to the computational method's order of accuracy, which is first-order. To show the latter, in the right graph of Figure 4 the time evolution of the relative air-mass error is also given for a grid and time step twice as fine. (The orders of accuracy of the oscillatory and linear error component - in going from $h=\frac{1}{40}$ to $h=\frac{1}{80}$ - appear to be 0.78 and 0.90 , respectively.)

\section{Conclusions}

To avoid large solution errors near interfaces, a problem for many conservative capturing methods, a simple ghost-fluid fix has been proposed. For density ratios of the order 1000 (typical water-air ratio) the simple ghost-fluid technique performs perfectly. Even the computation of fronts running into vacuum 

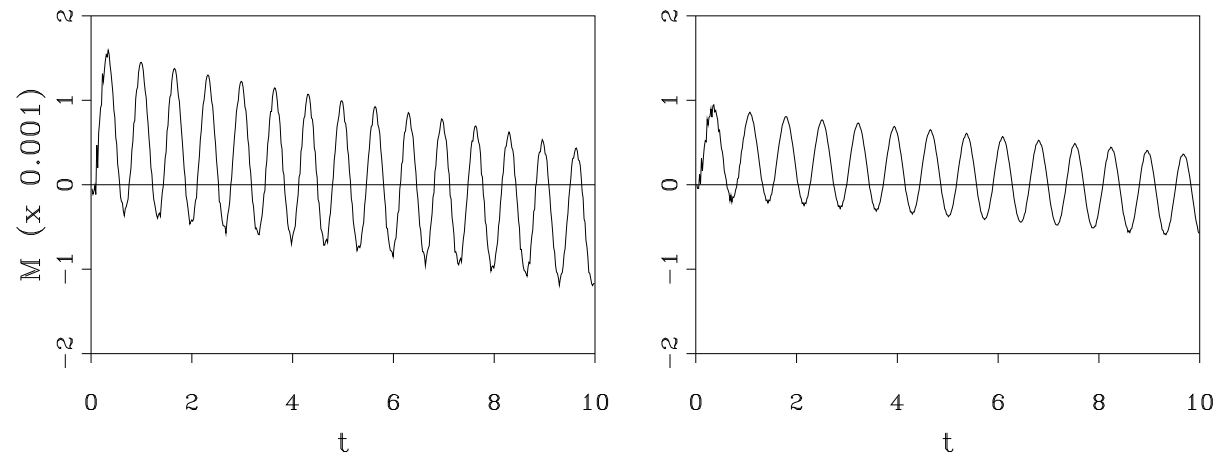

Fig. 4. Time evolution of relative error in total mass of air in closed tube, left: $h=\frac{1}{40}$, right: $h=\frac{1}{80}$

is expected to be possible. Extension of the method to higher-order accuracy is straightforward through the use of, e.g., a MUSCL approach and a multi-stage time integrator. Concerning the extension to higher dimensions, no principal difficulties exist.

\section{Acknowledgement}

This research was supported by the Dutch Technology Foundation STW.

\section{References}

1. R. Abgrall: J. Comput. Phys. 125, 150 (1996)

2. R. Abgrall, S. Karni: J. Comput. Phys. 169, 594 (2001)

3. R.P. Fedkiw, T. Aslam, B. Merriman, S. Osher: J. Comput. Phys. 152, 457 (1999)

4. C.W. Hirt, B.D. Nichols: J. Comput. Phys. 39, 201 (1981)

5. P. Jenny, B. Müller, H. Thomann: J. Comput. Phys. 132, 91 (1997)

6. S. Karni: J. Comput. Phys. 112, 31 (1994)

7. S. Karni: SIAM J. Sci. Comput. 17, 1019 (1996)

8. B. Koren, M.R. Lewis, E.H. van Brummelen, B. van Leer: Report MAS-R0112, CWI, Amsterdam (2001)

9. R. Saurel, R. Abgrall: SIAM J. Sci. Comput. 21, 1115 (1999)

10. J.A. Sethian: Level Set Methods: Evolving Interfaces in Geometry, Fluid Mechanics, Computer Vision, and Materials Science (Cambridge University Press, Cambridge, 1996) 